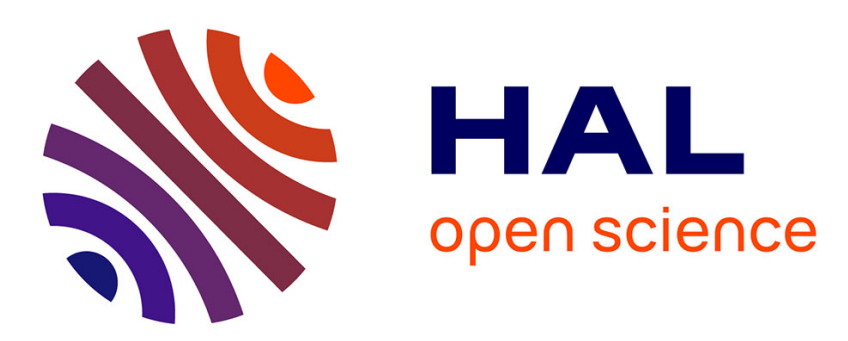

\title{
Convergence rates with singular parameterizations for solving elliptic boundary value problems in isogeometric analysis
}

Meng Wu, Yicao Wang, Bernard Mourrain, Boniface Nkonga, Changzheng Cheng

\section{To cite this version:}

Meng Wu, Yicao Wang, Bernard Mourrain, Boniface Nkonga, Changzheng Cheng. Convergence rates with singular parameterizations for solving elliptic boundary value problems in isogeometric analysis. Computer Aided Geometric Design, 2017, 52-53, pp.170-189. 10.1016/j.cagd.2017.02.006 . hal01276699v2

\section{HAL Id: hal-01276699 \\ https://hal.inria.fr/hal-01276699v2}

Submitted on 14 Nov 2016

HAL is a multi-disciplinary open access archive for the deposit and dissemination of scientific research documents, whether they are published or not. The documents may come from teaching and research institutions in France or abroad, or from public or private research centers.
L'archive ouverte pluridisciplinaire HAL, est destinée au dépôt et à la diffusion de documents scientifiques de niveau recherche, publiés ou non, émanant des établissements d'enseignement et de recherche français ou étrangers, des laboratoires publics ou privés. 


\title{
Convergence rates with singular parameterizations for solving elliptic boundary value problems in isogeometric analysis
}

\author{
Meng $\mathrm{Wu}^{\mathrm{a}}$, Yicao Wang ${ }^{\mathrm{b}}$, Bernard Mourrain ${ }^{\mathrm{c}}$, Boniface Nkonga ${ }^{\mathrm{d}}$, Changzheng \\ Cheng, ${ }^{\mathrm{a}, *}$ \\ ${ }^{a}$ School of Mathematics, Hefei University of Technology, P. R. China. \\ ${ }^{b}$ Department of Mathematics, Hohai University, P. R. China \\ ${ }^{c}$ Galaad2, Inria, Sophia Antipolis, France \\ ${ }^{d}$ Lab. J. A. Dieudonné, University of Nice, Nice, France
}

\begin{abstract}
In this paper, we present convergence rates for solving elliptic boundary value problems with singular parameterizations in isogeometric analysis. First, the approximation errors with the $L^{2}(\Omega)$-norm and the $H^{1}(\Omega)$-seminorm are estimated locally. The impact of singularities is considered in this framework. Second, the convergence rates for solving PDEs with singular parameterizations are discussed. These results are based on a weak solution space that contains all of the weak solutions of elliptic boundary value problems with smooth coefficients. For the smooth weak solutions obtained by isogeometric analysis with singular parameterizations and the finite element method, both are shown to have the optimal convergence rates. For non-smooth weak solutions, the optimal convergence rates are reached by setting proper singularities of a controllable parameterization, even though convergence rates are not optimal by finite element method, and the convergence rates by isogeometric analysis with singular parameterizations are better than the ones by the finite element method.
\end{abstract}

Keywords: singular parameterizations, convergence rates, elliptic boundary value problems, isogeometric analysis

\section{Introduction}

Isogeometric analysis (IGA) [1] is a numerical method to solve geometric partial differential equations. The theory of approximation and error estimates has been developed in $[2,3]$, where the theory relies on the regularity of parameterizations, i.e., there are no singularities of the underlaying parameterizations.

${ }^{*}$ Corresponding author at: Hefei University of Technology, No. 193, Tunxi Road, Hefei, Anhui Prov., 230009, P. R. China.

Email addresses: meng.wu@hfut.edu.cn, wumeng@mail.ustc.edu.cn (Meng Wu), yicwang@hhu.edu.cn (Yicao Wang), Bernard.Mourrain@inria.fr (Bernard Mourrain), boniface.nkonga@unice.fr (Boniface Nkonga), changzheng.cheng@hfut.edu.cn (Changzheng Cheng) 
Although singularities can be avoided by using some constraints $[4,5,6]$ in some cases, some singularities may stem essentially from the geometry or topology of the physical domain and cannot be avoided. For instance, in [7], for a topologically circular domain, the parameterization is singular at 'a converging point'. In [8], singularities appear at extraordinary vertices if the parameterization is asked to have $C^{1}$ continuity. In this paper, we present the convergence rates for solving elliptic boundary value problems with singular parameterizations.

Recently, there are works focused on singular parameterizations in the IGA. In $[9,10]$, the regularity of test functions on physical domains is discussed. Afterwards, the authors continue to work on this topic. One of the authors presents results on the approximation properties of isogeometric analysis function spaces in [11] and construct smooth isogeometric function spaces [12] on a singularly parameterized domain. In addition, in enriched isogeometric analysis (EIGA) [13, 14], the authors introduce a mapping technique which is an additional singular mapping patch used to generate singular functions in physical domains with cracks and/or corners. In [14], the error estimates of this mapping technique on an elliptic boundary value problem are presented. So far, singular parameterizations are discussed independently for solving elliptic boundary value problems with smooth weak solutions or non-smooth weak solutions. In the present work, the results are based on a weak solution space $\mathcal{F}(\Omega)$ which contains all possible weak solutions of an elliptic boundary problem with smooth coefficients. The main contributions are listed in the following.

1. For functions in $\mathcal{F}(\Omega)$, we present the approximation estimates with the $L^{2}(\Omega)$ norm and the $H^{1}(\Omega)$-seminorm. Different from previous work, approximation errors are estimated locally, i.e., the impact of the singularities of the parameterization is considered.

2. Based on the approximation error estimates, we discuss the convergence rates of solving elliptic boundary problems with singular parameterizations. By introducing a controllable singular parameterization, optimal convergence rates are reached by setting singularities even if the optimal convergence rates cannot be reached by the finite element method (FEM). Moreover, convergence rates are discussed in the IGA with singular parameterizations.

The paper is structured as follows. Approximation error estimates are presented in Section 2. After that, in Section 3, we discuss convergence rates for solving elliptic boundary problems based on the error estimates of Section 2. Finally, in Section 4, we conclude this paper with a discussion of possibilities for future works.

\section{Approximation Error Estimates of Singular parameterizations}

In this section, as background, parameterizations and singularities of parameterizations are introduced in Section 2.1. Then, in Section 2.2, by Weyl's lemma, a set of functions $\mathcal{F}(\Omega)$ called a weak solution space is introduced, where $\mathcal{F}(\Omega)$ contains all the weak solutions of elliptic boundary value problems with smooth coefficients on a phys-

ical domain $\Omega$. In addition, a Hermite projection $\Pi_{\mathscr{H}_{h}^{p}}^{\hat{h}}$ is introduced as a preparation 
for presenting the $L^{2}(\Omega)$-norm and $H^{1}(\Omega)$-seminorm approximation error estimates of singular parameterizations in Theorems 2.3 and 2.4 of Section 2.3.

\subsection{Parameterizations and their singularities}

Definition 2.1. A parameterization $\mathscr{P}(s, t)$ is a map from a parametric domain to a physical domain, i.e.

$$
\begin{aligned}
\mathscr{P}: \Sigma & \longrightarrow \Omega ; \\
(s, t) & \longrightarrow(x(s, t), y(s, t)) .
\end{aligned}
$$

For discussing the convergence rate for solving elliptic boundary value problems, the parameterization $\mathscr{P}(s, t)$ satisfies the following properties:

(1). $\mathscr{P}(s, t)$ is a one-to-one map.

(2). To solve the elliptic boundary problem, $\mathscr{P}(s, t)$ satisfies the $H^{1}$ integrability assumption [9], i.e., for any $f(s, t)$ in a given isogeometric function space, $f \circ \mathscr{P}^{-1}(x, y) \in$ $H^{1}(\Omega)$. If $\mathscr{P}(s, t)$ satisfies the $H^{1}$ integrability assumption, we call it an $H^{1}$-parameterization. (3). $\mathscr{P}(s, t)$ preserves the geometry of $\Omega$, i.e., $\left.\mathscr{P}\right|_{\partial \Sigma}=\partial \Omega$ and $\mathscr{P}\left(\Sigma^{o}\right)=\Omega^{o}$, where $\Sigma^{o}, \Omega^{o}$ are the interior domain of $\Sigma$ and $\Omega$ respectively.

Definition 2.2. The Jacobian of $\mathscr{P}(s, t)$ is defined by

$$
J(s, t)=\frac{\partial x}{\partial s} \frac{\partial y}{\partial t}-\frac{\partial x}{\partial t} \frac{\partial y}{\partial s}
$$

If $J\left(s_{0}, t_{0}\right)=0$, then $\left(s_{0}, t_{0}\right)$ is called a singularity of $\mathscr{P}(s, t)$.

In the following, we suppose that $J(s, t) \geq 0$ and the singularities are isolated. Furthermore, at a singularity $\left(s_{0}, t_{0}\right), \mathscr{P}(s, t)$ satisfies

$$
\frac{\partial x}{\partial s}\left(s_{0}, t_{0}\right)=0, \frac{\partial y}{\partial s}\left(s_{0}, t_{0}\right)=0, \frac{\partial x}{\partial t}\left(s_{0}, t_{0}\right)=0, \frac{\partial y}{\partial t}\left(s_{0}, t_{0}\right)=0 .
$$

In Example 2.1, there is an $H^{1}$-parameterization of a geometry with a smooth boundary.

Example 2.1 (A geometry with a smooth boundary). In this example, parameterizations of a geometry with a smooth boundary are presented. The parametric domain $\Sigma$ is $[0,1] \times[0,1]$. To fit the smooth boundary in Figure $1(b), \mathscr{P}(s, t)$ is singular at the corner points $P_{1}(0,0), P_{2}(1,0), P_{3}(0,1), P_{4}(1,1)$. Although the tangents of the $s$-curve and the t-curve are degenerate, the limits of the unit tangents of the $s$-curve and the t-curve at these points are collinear. 


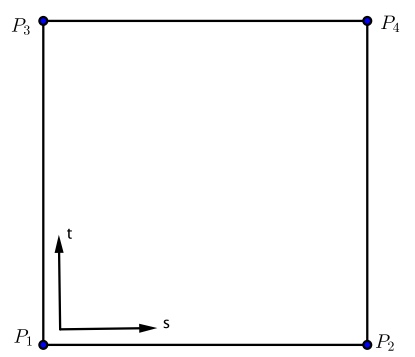

(a) $\Sigma$

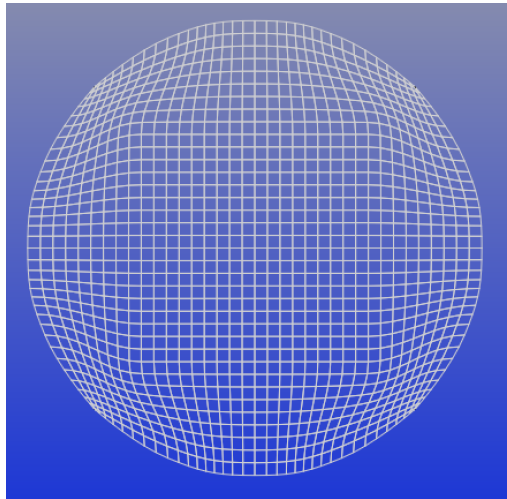

(b) $\mathscr{P}(s, t)$

Figure 1: A parameterization of a geometry with a smooth boundary

\subsection{Weak solution spaces and Hermite Interpolation}

Consider the regularity of weak solutions of an elliptic boundary value problem:

$$
\begin{aligned}
& -\nabla \cdot(p(x, y) \nabla u)+q(x, y) u=f(x, y) \text { in } \Omega, \\
& u=0 \text { on } \partial \Omega .
\end{aligned}
$$

Based on Weyl's lemma [15], if $p(x, y), q(x, y), f(x, y)$ are analytic at the interior points of $\Omega$, (i.e., on a neighborhood of an interior point $P$, their Taylor expansions converge to themselves), the weak solution $u$ is analytic at these points. If $\partial \Omega$ is analytic, $u$ is analytic on $\Omega$. For $\Omega$ with non-smooth boundaries, non-analytic points are among these non-smooth boundary points.

In the following, there is an example of a weak solution of (1).

Example 2.2. Let $\Omega$ be the physical domain shown in Figure 2. There is a corner $P$. The weak solution $u$ of the elliptic boundary value problem $(1)$ with $p(x, y)=1$ and $q(x, y)=0$ is non-analytic at $P$.

If $\theta=\alpha \pi$, in [15], then the weak solution $u$ around the corner $P$ is

$$
u(r, \theta)=\sum_{j=1}^{\infty} \alpha_{j} r^{v_{j}} \phi_{j}(\theta)+\sum_{j=1}^{\infty} \sum_{\ell=0}^{\infty} f_{j \ell}\left[(\ell+2)^{2}-v_{j}^{2}\right]^{-1} \phi_{j}(\theta) r^{\ell+2},
$$

where

$$
\begin{aligned}
& \phi_{j}(\theta)=\sqrt{\frac{2}{\alpha \pi}} \sin v_{j} \theta, v_{j}=j / \alpha ; f_{j}(r)=\int_{0}^{\alpha \pi} f \phi_{j}(\theta) d \theta, \quad f_{j}(r)=\sum f_{j \ell} r^{\ell}, \\
& \Omega_{0}=\left\{(r, \theta) \mid 0<r<r_{0}, 0<\theta<\alpha \pi\right\} \subset \Omega
\end{aligned}
$$

i.e., it has a singularity of type $O\left(r^{1 / \alpha}\right)$. Except for at the point $P$, the solution $u$ is analytic. In this case, if $1 / \alpha \notin \mathbb{N}$, then $u \in H^{\sigma}(\Omega)$ where $1<\sigma<2$ and $r=\sqrt{x^{2}+y^{2}}$. 


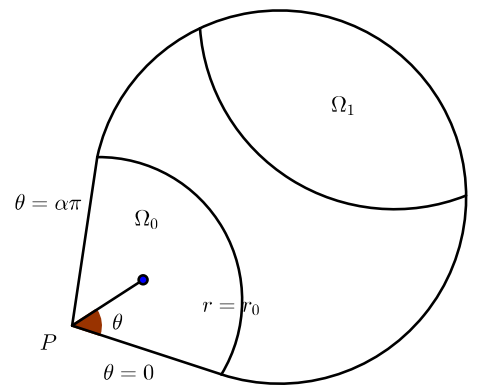

Figure 2: A physical domain $\Omega$ with a corner

To describe all the weak solutions on $\Omega$, we define a function space called a weak solution space,

$$
\mathcal{F}(\Omega)=\left\{f: \Omega \longrightarrow \mathbb{R}: f(x, y) \text { is analytic on } \Omega^{o}\right\} \subset C^{\infty}\left(\Omega^{o}\right) .
$$

The weak solutions on $\Omega$ of the elliptic boundary value problem are in $\mathcal{F}(\Omega)$. Let

$$
\mathscr{P}: \Sigma \longrightarrow \Omega
$$

be a parameterization of $\Omega$, where $\mathscr{P}(s, t) \in C^{\ell}(\Sigma)$. Thus,

$$
\mathcal{F} \circ \mathscr{P} \subset C^{\ell}\left(\Sigma^{o}\right) .
$$

Let $\mathcal{Q}_{h}$ be a regular mesh, i.e., it is a rectangular mesh and the valences of its interior vertices are 4 , defined on a parametric domain $\Sigma$, where $h$ is the maximum of the diameters of its cells. In Figure 3, there are three regular meshes.
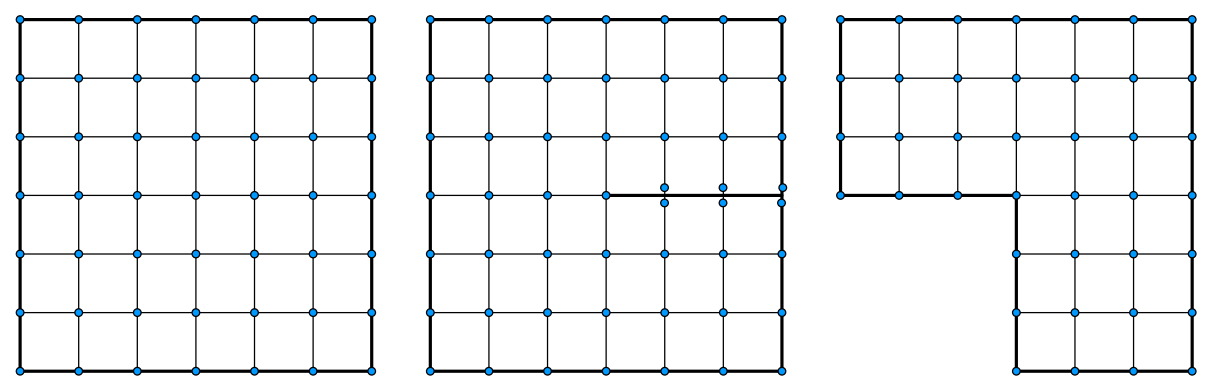

Figure 3: Regular Meshes $\mathcal{Q}_{h}$, where the bold edges are boundaries.

$\mathscr{H}_{h}^{p}\left(\mathcal{Q}_{h}\right)$ is the spline space with bidegree $(p, p)$ defined on $\mathcal{Q}_{h}$, and $\mathscr{H}_{h}^{p}\left(\mathcal{Q}_{h}\right) \subset$ $C^{\ell}(\Sigma)$, where $p=2 \ell+1$. In the following, we define an interpolation projection from $\mathcal{F}(\Omega)$ to $\mathscr{H}_{h}^{p}\left(\mathcal{Q}_{h}\right)$. 
Associated to each vertex $v_{k} \in \mathcal{V}\left(\mathcal{Q}_{h}\right)$, we can construct $(\ell+1)^{2}$ Hermite functions $\left\{H_{i, j}^{v_{k}}(s, t): i, j=0,1, \cdots, \ell\right\}$, where $H_{i, j}^{v_{k}}(s, t)$ satisfies

$$
\frac{\partial^{r_{1}+r_{2}} H_{i, j}^{v_{k}}\left(v_{l}\right)}{\partial s^{r_{1}} \partial t^{r_{2}}}=\delta_{k, l} \delta_{r_{1}, i} \delta_{r_{2}, j}
$$

where $r_{1}, r_{2}=0,1,2, \cdots, \ell, v_{k}, v_{l} \in \mathcal{V}\left(\mathcal{Q}_{h}\right), \delta_{i, j}=0$ if $i \neq j$, otherwise $\delta_{i, j}=1$. Following [16], it is easy to prove that $\cup_{v_{k} \in \mathcal{V}\left(\mathcal{Q}_{h}\right)}\left\{H_{i, j}^{v_{k}}(s, t): i, j=0,1, \cdots, \ell\right\}$ is a set of basis functions of $\mathscr{H}_{h}^{p}\left(\mathcal{Q}_{h}\right)$. Define a $\mathcal{V}\left(\mathcal{Q}_{h}\right)$-based Hermite projection

$$
\Pi_{\mathscr{H}_{h}^{p}}^{\mathcal{V}\left(\mathcal{Q}_{h}\right)}: C^{\ell}(\bar{\Sigma}) \longrightarrow \mathscr{H}_{h}^{p}\left(\mathcal{Q}_{h}\right)
$$

such that

$$
\Pi_{\mathscr{H}_{h}^{p}}^{\mathcal{V}\left(\mathcal{Q}_{h}\right)}(f(s, t))=\sum_{v \in \mathcal{V}\left(\mathcal{Q}_{h}\right)} \frac{\partial^{i+j} f(v)}{\partial s^{i} \partial t^{j}} H_{i, j}^{v}(s, t) .
$$

However, for the weak solution space $\mathcal{F}(\Omega), \mathcal{F} \circ \mathscr{P} \subset C^{\ell}\left(\Sigma^{o}\right)$, in other words, the Hermite projection (4) is not well defined on the boundary vertices of $\mathcal{Q}_{h}$. To solve this problem, for each boundary vertex $v^{b}$ of $\mathcal{Q}_{h}$, there is a point $q \in \Sigma^{o}$ such that we can construct a set of linear independent splines $\left\{H_{i, j}^{q}(s, t): i, j=0,1, \cdots, \ell\right\}$ associated with $q$. In addition, $\left\{H_{i, j}^{q}(s, t): i, j=0,1, \cdots, \ell\right\}$ is linearly equivalent to $\left\{H_{i, j}^{v^{b}}(s, t): i, j=0,1, \cdots, \ell\right\}$, i.e., for each $H_{i, j}^{q}(s, t)$, it is a linear combination of $\left\{H_{i, j}^{v^{b}}(s, t): i, j=0,1, \cdots, \ell\right\}$; the reverse is also true. Here we present this theorem.

Theorem 2.1. Let $\Sigma$ be a parametric domain. $\mathcal{Q}_{h}$ is a regular mesh on $\Sigma$. For each boundary vertex $v_{k}^{b} \in \mathcal{V}\left(\mathcal{Q}_{h}\right)$, there is a point $q_{k} \in \Sigma^{o}$ such that $\left\{H_{i, j}^{q_{k}}(s, t)\right.$ : $i, j=0,1, \cdots, \ell\}$ are linearly equivalent to $\left\{H_{i, j}^{v_{k}^{b}}(s, t): i, j=0,1, \cdots, \ell\right\}$, and $H_{i, j}^{q_{k}}(s, t)$ satisfies $\forall q_{l} \in \mathcal{V}\left(\mathcal{Q}_{h}\right) \cup\left\{q_{k}\right\}-\left\{v_{k}^{b}\right\}$

$$
\frac{\partial^{r_{1}+r_{2}} H_{i, j}^{q_{k}}\left(q_{l}\right)}{\partial s^{r_{1}} \partial t^{r_{2}}}=\delta_{k, l} \delta_{i r_{1}} \delta_{j r_{2}}
$$

Proof. The proof is divided into two parts. In the first part, we prove the existence of $q_{k}$ and construct $H_{i, j}^{q_{k}}(s, t)$. In the second part, prove the linear relationship between $\left\{H_{i, j}^{q_{k}}(s, t): i, j=0,1, \cdots, \ell\right\}$ and $\left\{H_{i, j}^{v_{k}^{b}}(s, t): i, j=0,1, \cdots, \ell\right\}$.

1. The existence of $q_{k}$ : Consider the matrix

$$
H^{v_{k}^{b}}(s, t)=\left(\frac{\partial^{r_{1}+r_{2}} H_{i, j}^{v_{k}^{b}}}{\partial s^{r_{1}} \partial t^{r_{2}}}(s, t)\right)_{(\ell+1)^{2} \times(\ell+1)^{2}}
$$

Its rows and columns are arranged by the $(i, j)$-order and $\left(r_{1}, r_{2}\right)$-order, respectively, where the $(i, j)$-order and the $\left(r_{1}, r_{2}\right)$-order are $(0,0) \prec(1,0) \prec \cdots \prec(\ell, 0) \prec(0,1) \prec$ 
$(1,1) \prec \cdots, \prec(\ell, 1), \prec \cdots, \prec(0, \ell) \prec(1, \ell) \prec \cdots \prec(\ell, \ell)$. Then, on a cell $C$ of $\mathcal{Q}_{h}$ with $v_{k}^{b}$ as one of its corner point, $\left.\operatorname{det} H^{v_{k}^{b}}(s, t)\right|_{C}$ is a polynomial and

$$
\operatorname{det} H^{v_{k}^{b}}\left(v_{k}^{b}\right)=1 \neq 0
$$

because $H^{v_{k}^{b}}\left(v_{k}^{b}\right)$ is an identity matrix. Thus, there is a neighborhood of $v_{k}^{b}, U\left(v_{k}^{b}\right)$, such that for any $q \in U\left(v_{k}^{b}\right) \cap C$,

$$
\operatorname{det} H^{v_{k}^{b}}\left(v_{k}^{b}\right) \neq 0 .
$$

Take $q_{k} \in U\left(v_{k}^{b}\right) \cap C$ and construct the following functions in $\mathscr{H}_{h}^{p}\left(\mathcal{Q}_{h}\right)$

$$
H_{i, j}^{q_{k}}(s, t)=\sum_{k, l=0}^{\ell} C_{k, l} H_{k, l}^{v_{k}^{b}}(s, t)
$$

and $\mathbf{C}=\left(C_{0,0}, C_{1,0}, \cdots, C_{\ell, 0}, \cdots, C_{0, \ell}, \cdots, C_{\ell, \ell}\right)^{T}$ satisfy

$$
\mathbf{C}=\left(H^{v_{k}^{b}}\left(q_{k}\right)\right)^{-1} \mathbf{e}_{i, j},
$$

where $\left(H^{v_{k}^{b}}\left(q_{k}\right)\right)^{-1}$ exists because det $H^{v_{k}^{b}}\left(q_{k}\right) \neq 0$; Additional, $\mathbf{e}_{i, j}=(0, \cdots, 0,1,0, \cdots, 0)^{T}$ is a column vector, i.e., the $(i, j)$-element is 1 associated with the $(i, j)$-order. Other elements are zeros. Computing directly, we obtain

$$
\frac{\partial^{r_{1}+r_{2}} H_{i, j}^{q_{k}}\left(q_{l}\right)}{\partial s^{r_{1}} \partial t^{r_{2}}}=\delta_{k, l} \delta_{i, r_{1}} \delta_{j, r_{2}}, \forall q_{l} \in \mathcal{V}\left(\mathcal{Q}_{h}\right) \cup\left\{q_{k}\right\}-\left\{v_{k}^{b}\right\} .
$$

2. The linear relationship: Denote

$$
\begin{aligned}
& \mathbf{H}^{q_{k}}(s, t)=\left(H_{0,0}^{q_{k}}(s, t), \cdots, H_{i, j}^{q_{k}}(s, t), \cdots, H_{\ell, \ell}^{q_{k}}(s, t)\right) \\
& \mathbf{H}^{v_{k}^{b}}(s, t)=\left(H_{0,0}^{v_{k}^{b}}(s, t), \cdots, H_{i, j}^{v_{k}^{b}}(s, t), \cdots, H_{\ell, \ell}^{v_{k}^{b}}(s, t)\right)
\end{aligned}
$$

By (6),

$$
\mathbf{H}^{q_{k}}(s, t)=\mathbf{H}^{v_{k}^{b}}(s, t)\left(H^{v_{k}^{b}}\left(q_{k}\right)\right)^{-1},
$$

i.e.,

$$
\mathbf{H}^{q_{k}}(s, t) H^{v_{k}^{b}}\left(q_{k}\right)=\mathbf{H}^{v_{k}^{b}}(s, t) .
$$

Thus $\left\{H_{i, j}^{q_{k}}(s, t): i, j=0,1, \cdots, \ell\right\}$ and $\left\{H_{i, j}^{v_{k}^{b}}(s, t): i, j=0,1, \cdots, \ell\right\}$ are linearly equivalent.

Based on this theorem, by replacing each boundary vertex $v_{k}^{b} \in \mathcal{V}\left(\mathcal{Q}_{h}\right)$ by $q_{k} \in \Sigma^{o}$, another set of interpolation points $\hat{A}$ of $\mathscr{H}_{h}^{p}\left(\mathcal{Q}_{h}\right)$ is introduced and $\hat{A} \subset \Sigma^{o}$. Thus, an $\hat{A}$-based Hermite projection $\Pi_{\mathscr{H}_{h}^{p}}^{\hat{A}}$ can be defined such that

$$
\Pi_{\mathscr{H}_{h}^{p}}^{\hat{\hat{p}}}: \mathcal{F} \circ \mathscr{P} \longrightarrow \mathcal{H}_{h}^{p}\left(\mathcal{Q}_{h}\right)
$$


where

$$
\Pi_{\mathscr{H}_{h}^{p}}^{\hat{A}}(f)=\sum_{q_{k} \in \hat{A}} \frac{\partial^{i+j} f\left(q_{k}\right)}{\partial s^{i} \partial t^{j}} H_{i, j}^{q_{k}}(s, t) .
$$

By the B-net method [16], the Bezier coordinates around $q_{k}$ are equivalent to the ones around $v_{k}^{b}$ and they are independent of the Beźier coordinates around the other vertices of $\hat{A}$. Moreover, for each polynomial $p(s, t)$ with bidegree $(k, k)$

$$
\Pi_{\mathscr{H}_{h}^{p}}^{\mathcal{V}\left(\mathcal{Q}_{h}\right)}(p(s, t))=p(s, t)
$$

where $k \leq p$. Thus, we have

\section{Lemma 2.2.}

$$
\Pi_{\mathscr{H}_{h}^{p}}^{\hat{A}}(p(s, t))=p(s, t), p(s, t) \in \mathbb{P}_{[k, k]} .
$$

where $k \leq p, \mathbb{P}_{[k, k]}$ is a polynomial space with bidegree $(k, k)$.

Induced by $\mathscr{H}_{h}^{p}\left(\mathcal{Q}_{h}\right)$ and $\mathscr{P}(s, t)$, one can define a projection

$$
\begin{aligned}
\Pi_{\mathcal{V}_{h}}^{\hat{A}}: \mathcal{F} & \longrightarrow \mathcal{V}_{h} \\
& u(x, y) \rightarrow \Pi_{\mathscr{H}_{h}^{p}}^{\hat{A}}(u \circ \mathscr{P}(s, t)) \circ \mathscr{P}^{-1},
\end{aligned}
$$

where

$$
\mathcal{V}_{h}=\mathscr{H}_{h}^{p}\left(\mathcal{Q}_{h}\right) \circ \mathscr{P}^{-1}=\operatorname{span}\left\{H_{i, j}^{q}(s, t): q \in \hat{A}, i, j=0,1, \cdots, \ell\right\}
$$

and $\left\{H_{i, j}^{q}(s, t): q \in \hat{A}, i, j=0,1, \cdots, \ell\right\}$ are the basis functions of $\mathscr{H}_{h}^{p}\left(\mathcal{Q}_{h}\right)$. In the next section, we begin to measure the $L^{2}(\Omega)$-norm and the $H^{1}(\Omega)$-seminorm approximation error estimates of singular parameterizations.

\section{3. $L^{2}(\Omega)$-norm and $H^{1}(\Omega)$-seminorm approximation error estimates of singular pa- rameterizations}

To measure approximation error estimates of singular parameterizations, we introduce some symbols.

\section{Classifying cells of $\mathcal{Q}_{h}$ :}

$C_{s}$ is a cell of $\mathcal{Q}_{h}$ on $\Sigma$, where there is a singularity of $\mathscr{P}$ on it. Cells not of this type are called regular cells of $\Sigma$ and are denoted by $C_{r}$.

$$
\Sigma_{s}=\cup C_{s} \text {, and } \Sigma_{r}=\cup C_{r} .
$$

\section{The definition of norms:}

$\forall u \in \mathcal{F}(\Omega) \cap W^{m, p}(\Omega)$, the norms and seminorms follow the classical definitions in $W^{m, p}(\Omega)$, where $W^{m, p}(\Omega)$ is the Sobolev space, i.e., for $1 \leq p<\infty$,

$$
\|u\|_{m, p, \Omega}=\left(\sum_{|\alpha| \leq m} \int_{\Omega}\left|\partial^{\alpha} u\right|^{p} d \Omega\right)^{\frac{1}{p}},|u|_{m, p, \Omega}=\left(\sum_{|\alpha|=m} \int_{\Omega}\left|\partial^{\alpha} u\right|^{p} d \Omega\right)^{\frac{1}{p}}
$$


and for $p=\infty$,

$$
\begin{aligned}
& \left.|| u\right|_{m, \infty, \Omega}=\max _{|\alpha| \leq m}\left\{\text { ess. } \sup \left|\partial^{\alpha} u(x, y)\right|:(x, y) \in \Omega\right\} \\
& |u|_{m, \infty, \Omega}=\max _{|\alpha|=m}\left\{\text { ess. } \sup \left|\partial^{\alpha} u(x, y)\right|:(x, y) \in \Omega\right\}
\end{aligned}
$$

where $\alpha=\left(\alpha_{1}, \alpha_{2}\right), \partial^{\alpha}=\partial_{1}^{\alpha_{1}} \partial_{2}^{\alpha_{2}},|\alpha|=\alpha_{1}+\alpha_{2}$. Especially, when $p=2$,

$$
\|u\|_{L^{2}(\Omega)}=\|u\|_{0,2, \Omega} ;\|u\|_{H^{k}(\Omega)}=\|u\|_{k, 2, \Omega}(k \geq 1) ;|u|_{k, 2, \Omega}=|u|_{H^{k}(\Omega)} .
$$

In the following, the approximation error estimates with the $L^{2}(\Omega)$-norm and the $H^{1}(\Omega)$-seminorm are presented.

Theorem 2.3. Let $\Sigma$ and $\Omega$ be a parametric domain and a physical domain.

$$
\mathscr{P}: \Sigma \longrightarrow \Omega
$$

is a parameterization of $\Omega . u(x, y) \in \mathcal{F}(\Omega) \cap L^{2}(\Omega)$ is a bounded function on $\Omega$ and $\left.u \circ \mathscr{P}\right|_{\Sigma_{r}} \in H^{k+1}\left(\Sigma_{r}\right),\left.u \circ \mathscr{P}\right|_{\Sigma_{s}} \in H^{k^{\prime}+1}\left(\Sigma_{s}\right)$. Then,

$$
\| u-\left.\Pi_{\mathcal{V}_{h}}^{\hat{A}} u\right|_{L^{2}(\Omega)} \leq K_{1} h^{k+1}|u \circ \mathscr{P}|_{H^{k+1}\left(\Sigma_{r}\right)}+K_{2} h^{\sigma / 2+k^{\prime}+1}|u \circ \mathscr{P}|_{H^{k^{\prime}+1}\left(\Sigma_{s}\right)},
$$

where $h$ is the maximum diameter of the cells of the parametric mesh $\mathcal{Q}_{h}$ on $\Sigma, \mathcal{V}_{h}=$ $\mathscr{H}_{h}^{p} \circ \mathscr{P}^{-1}\left(p \geq \min \left\{k, k^{\prime}\right\}\right) .\left.J\right|_{C_{s}} \sim h^{\sigma_{C_{s}}}, \sigma=\min \left\{\sigma_{C_{s}}\right\}, K_{1}=K_{1}\left(\mathscr{P}, k, \Pi_{\mathscr{H}_{h}^{p}}^{\hat{A}}\right)$ and $K_{2}=K_{2}\left(\mathscr{P}, k^{\prime}, \Pi_{\mathscr{H}_{h}^{p}}^{\hat{A}}\right)$, i.e., $K_{1}$ and $K_{2}$ are independent of the mesh size $h$ and $u(x, y)$, but are dependent on the regularity of $u \circ \mathscr{P}, \Pi_{\mathscr{H}_{h}^{p}}^{\hat{A}}$ and its parameterization $\mathscr{P}(s, t)$.

\section{Proof.}

$$
\begin{aligned}
\| u & -\Pi_{\mathcal{V}_{h}}^{\hat{A}} u \|_{L^{2}(\Omega)}^{2}=\sum_{C} \int_{C}\left(u \circ \mathscr{P}-\Pi_{\mathscr{H}_{h}^{p}}^{\hat{A}}(u \circ \mathscr{P})\right)^{2}|J| d s d t \\
& =\sum_{C_{r}} \int_{C_{r}}\left(u \circ \mathscr{P}-\Pi_{\mathscr{H}_{h}^{p}}^{\hat{A}}(u \circ \mathscr{P})\right)^{2}|J| d s d t+\sum_{C_{s}} \int_{C_{s}}\left(u \circ \mathscr{P}-\Pi_{\mathscr{H}_{h}^{p}}^{\hat{A}}(u \circ \mathscr{P})\right)^{2}|J| d s d t \\
& \leq K_{1}^{1} \sum_{C_{r}} \int_{C_{r}}\left(u \circ \mathscr{P}-\Pi_{\mathscr{H}_{h}^{p}}^{\hat{A}}(u \circ \mathscr{P})\right)^{2} d s d t+K_{2}^{1} h^{\sigma} \sum_{C_{s}} \int_{C_{s}}\left(u \circ \mathscr{P}-\Pi_{\mathscr{H}_{h}^{p}}^{\hat{A}}(u \circ \mathscr{P})\right)^{2} d s d t, \\
& =K_{1}^{1} \sum_{C_{r}}\left\|u \circ \mathscr{P}-\Pi_{\mathscr{H}_{h}^{p}}^{\hat{A}}(u \circ \mathscr{P})\right\|_{L^{2}\left(C_{r}\right)}^{2}+K_{2}^{1} h^{\sigma} \sum_{C_{s}}\left\|\left(u \circ \mathscr{P}-\Pi_{\mathscr{H}_{h}^{p}}^{\hat{A}}(u \circ \mathscr{P})\right)\right\|_{L^{2}\left(C_{s}\right)}^{2},
\end{aligned}
$$

where $K_{1}^{1}$ and $K_{2}^{1}$ are only dependent on $\mathscr{P}$.

Based on Lemma 2.2,

$$
\Pi_{\mathscr{H}_{h}^{p}}^{\hat{A}} p(s, t)=p(s, t), \forall p \in \mathbb{P}_{[k, k]} \text { and } \Pi_{\mathscr{H}_{h}^{p}}^{\hat{A}} p(s, t)=p(s, t), \forall p \in \mathbb{P}_{\left[k^{\prime}, k^{\prime}\right]}
$$

because $k, k^{\prime} \leq p$. Thus,based on Theorem 5 in [19] on each cell,

$$
\begin{aligned}
& \left\|u \circ \mathscr{P}-\Pi_{\mathscr{H}_{h}^{p}}^{\hat{A}}\right\|_{L^{2}(C)} \leq K_{1}^{2}|u \circ \mathscr{P}|_{H^{k+1}(C)} h^{k+1}, \text { if } C \text { is a regular cell; } \\
& \left\|u \circ \mathscr{P}-\Pi_{\mathscr{H}_{h}^{p}}^{\hat{A}}\right\|_{L^{2}(C)} \leq K_{1}^{2}|u \circ \mathscr{P}|_{H^{k^{\prime}+1}(C)} h^{k^{\prime}+1}, \text { if } C \text { is a irregular cell, }
\end{aligned}
$$


where $\left.u \circ \mathscr{P}\right|_{C_{r}} \in H^{k+1}\left(C_{r}\right)$ and $\left.u \circ \mathscr{P}\right|_{C_{s}} \in H^{k^{\prime}+1}\left(C_{s}\right), K_{1}^{2}, K_{2}^{2}$ are only dependent on $k, k^{\prime}, \Pi_{\mathscr{H}_{h}^{p}}^{\hat{A}}$. Thus, we obtain the error bound (7), where $K_{1}, K_{2}$ are only dependent on $\mathscr{P}, k, \Pi_{\mathscr{H}_{h}^{p}}^{\hat{A}}$ and $\mathscr{P}, k^{\prime}, \Pi_{\mathscr{H}_{h}^{p}}^{\hat{A}}$, respectively.

Theorem 2.4. Let $u \in \mathcal{F}(\Omega) \cap H^{1}(\Omega)$ and $\mathcal{F} \circ \mathscr{P} \subset W_{\infty}^{k+1}(\Sigma)$ (for $p \geq k \geq 0$ ). Then

$$
\left|u-\Pi_{\mathcal{V}_{h}}^{\hat{A}} u\right|_{H^{1}(\Omega)} \leq C h^{k}|u \circ \mathscr{P}|_{k+1, \infty, \Sigma}, \forall u \in \mathcal{F} \circ \mathscr{P},
$$

where $C=C\left(\mathscr{P}, k, \Pi_{\mathcal{V}_{h}}^{\hat{A}}\right)$ and $\mathcal{V}_{h}=\mathscr{H}_{h}^{p} \circ \mathscr{P}^{-1},(p \geq 1)$.

\section{Proof.}

$$
\begin{aligned}
\mid u- & \left.u_{h}\right|_{H^{1}(\Omega)} ^{2}=\int_{\Omega}\left[\left(\frac{\partial\left(u-\Pi_{\mathcal{V}_{h}}^{\hat{A}} u\right)}{\partial x}\right)^{2}+\left(\frac{\partial\left(u-\Pi_{\mathcal{V}_{h}}^{\hat{A}} u\right)}{\partial y}\right)^{2}\right] d x d y \\
= & \int_{\Sigma} \frac{\left(\frac{\partial E(s, t)}{\partial s} \frac{\partial y}{\partial t}-\frac{\partial E(s, t)}{\partial t} \frac{\partial y}{\partial s}\right)^{2}+\left(\frac{\partial x}{\partial s} \frac{\partial E(s, t)}{\partial t}-\frac{\partial x}{\partial t} \frac{\partial E(s, t)}{\partial s}\right)^{2}}{J} d s d t \\
& E(s, t)=u \circ \mathscr{P}-\Pi_{\mathscr{H}_{h}^{p}}^{\hat{A}}(u \circ \mathscr{P}) \\
\leq & \int_{\Sigma} \frac{\left(\frac{\partial E(s, t)}{\partial s}\right)^{2}\left(\frac{\partial y}{\partial t}\right)^{2}+\left(\frac{\partial E(s, t)}{\partial t}\right)^{2}\left(\frac{\partial y}{\partial s}\right)^{2}+\left(\frac{\partial x}{\partial s}\right)^{2}\left(\frac{\partial E(s, t)}{\partial t}\right)^{2}+\left(\frac{\partial x}{\partial t}\right)^{2}\left(\frac{\partial E(s, t)}{\partial s}\right)^{2}}{J} d s d t \\
\leq & |E(s, t)|_{1, \infty, \Sigma}^{2} \int_{\Sigma} \frac{\left(\frac{\partial x}{\partial s}\right)^{2}+\left(\frac{\partial y}{\partial s}\right)^{2}+\left(\frac{\partial x}{\partial t}\right)^{2}+\left(\frac{\partial y}{\partial t}\right)^{2}}{J} d s d t \\
= & |E(s, t)|_{1, \infty, \Sigma}^{2}\left(|s \circ \mathscr{P}-1|_{\mathbf{H}^{1}(\Omega)}+\left|t \circ \mathscr{P}^{-1}\right|_{\mathbf{H}^{1}(\Omega)}\right) \\
\leq & C_{1}\left|u \circ \mathscr{P}-\Pi_{\mathscr{H}_{h}^{p}}^{\hat{A}}(u \circ \mathscr{P})\right|_{1, \infty, \Sigma}^{2},
\end{aligned}
$$

where $C_{1}=C_{1}(\mathscr{P})$ because $\left|s \circ \mathscr{P}^{-1}\right|_{H^{1}(\Omega)}<\infty$ and $\left|t \circ \mathscr{P}^{-1}\right|_{H^{1}(\Omega)}<\infty$ by the $H^{1}$-parameterization $\mathscr{P}(s, t)$. Moreover, by $k \leq p$ and Lemma 2.2,

$$
\Pi_{\mathscr{H}_{h}^{p}}^{\hat{A}} p(s, t)=p(s, t), \forall p \in \mathbb{P}_{[k, k]} .
$$

Thus, by Theorem 5 in [19],

$$
\left|u \circ \mathscr{P}-\Pi_{\mathscr{H}_{h}^{p}}^{\hat{\hat{n}}}(u \circ \mathscr{P})\right|_{1, \infty, \Sigma} \leq \| u \circ \mathscr{P}-\left.\Pi_{\mathscr{H}_{h}^{p}}^{\hat{A}}(u \circ \mathscr{P})\right|_{1, \infty, \Sigma} \leq C_{2}|u \circ \mathscr{P}|_{k+1, \infty, \Sigma} h^{k}
$$

where $C_{2}=C_{2}\left(k, \Pi_{\mathscr{H}_{h}^{p}}^{\hat{A}}\right)$.

Thus, we obtain the result.

From the results in Theorems 2.3 and 2.4, the regularity of $u \circ \mathscr{P}$ on a parametric domain determines the approximation order. By the multivariate Faà di bruno formula [17], which is a formula for derivatives of a composite function, we obtain the following theorem. 
Theorem 2.5. If $u \in \mathcal{F}(\Omega) \cap H^{k}(\Omega)$ and on each cell of $\mathcal{Q}_{h} \mathscr{P}(s, t) \in C^{\infty} \times C^{\infty}$, then $u \circ \mathscr{P} \in H^{k^{\prime}}(\Sigma)$. If $u \in \mathcal{F}(\Omega) \cap W_{\infty}^{k}(\Omega)$, then $u \circ \mathscr{P} \in W_{\infty}^{k^{\prime}}(\Sigma)$, where $k^{\prime} \geq k$.

For instance, in Section 3.2, $u \circ \mathscr{P}(s, t)$ becomes smooth even if $u \in H^{\sigma}(\Omega)$ by controlling the singularities of $\mathscr{P}(s, t)$, where $1<\sigma<2$.

\section{Convergence Rates of Solving Elliptic Boundary Problems with Singular Parameterizations}

In this section, we discuss convergence rates of solving elliptic boundary problems with singular parameterizations. An elliptic boundary problem is a model problem, and we introduce it as a background in Section 3.1. To discuss the impact of singularities, the convergence rates are presented by a controllable singular parameterization in Section 3.2. Then, in Section 3.3, we discuss the convergence rate with a singular parameterization within the IGA framework.

\subsection{Model problem: an elliptic boundary problem}

For elliptic boundary value problems,

$$
\begin{aligned}
& -\Delta u=f \text { in } \Omega, \\
& u=0 \text { on } \partial \Omega,
\end{aligned}
$$

where $\Omega$ is a physical domain and $f$ is assumed to be analytic on $\bar{\Omega}$. Its weak form reads:

$$
\text { find } u \in \mathcal{V}: \quad a(u, v)=l(v), \quad \forall v \in \mathcal{V}
$$

where $\mathcal{V}=\left\{\omega \in H^{1}(\Omega):\left.\omega\right|_{\partial \Omega}=0\right\}$ is a Hilbert space endowed with the norm of $\mathcal{V}$. Moreover,

$$
a(u, v)=\int_{\Omega} \nabla v^{T} \nabla u d \Omega, \quad l(v)=\int_{\Omega} f v d \Omega
$$

where $a: \mathcal{V} \times \mathcal{V} \longrightarrow \mathbf{R}$ is a continuous, (strongly) coercive bilinear form and $l: \mathcal{V} \longrightarrow \mathbf{R}$ is a continuous linear functional. In the framework of the FEM, the approximate solution $u_{h}$ is obtained by solving the following problem:

$$
\text { find } u_{h} \in \mathcal{V}^{h}: \quad a\left(u_{h}, v_{h}\right)=l\left(v_{h}\right), \quad \forall v_{h} \in \mathcal{V}^{h} \text {, }
$$

where $\mathcal{V}^{h} \subset \mathcal{V}$ is a nontrivial and finite dimensional subspace.

Here, we collect the results of the a priori estimate from the FEM from [15]. Let $\Omega$ be a polygonal domain, $f \in L^{2}(\Omega)$, and $u_{h}$ be the FEM solution.

1. If the exact weak solution $u \in H^{\sigma}(\Omega)(\sigma \geq 2)$, then

$$
\left\|u-u_{h}\right\|_{L^{2}(\Omega)} \leq C h^{l+1}\|u\|_{H^{l+1}(\Omega)},\left\|u-u_{h}\right\|_{H^{1}(\Omega)} \leq C h^{l}\|u\|_{H^{l+1}(\Omega)},
$$


where $l=\min (p, \sigma-1)$.

2. If $u \in H^{\sigma}(\Omega)(1<\sigma<2)$, then the error bound is

$$
\left\|u-u_{h}\right\|_{L^{2}(\Omega)} \leq C\left(h^{l+\sigma-1}+h^{2 p}\right)\|u\|_{H^{l+1}(\Omega)}, l=\min (p, \sigma-1),
$$

where $p$ is the degree of the piecewise polynomial basis functions.

In the IGA, Equation (10) is computed on the parametric domain $\Sigma$, i.e.,

$$
\begin{gathered}
a\left(u_{h}, v_{h}\right)=\int_{\Sigma}\left(\frac{\partial\left(u_{h}, y\right)}{\partial(s, t)} \frac{\partial\left(v_{h}, y\right)}{\partial(s, t)}+\frac{\partial\left(x, u_{h}\right)}{\partial(s, t)} \frac{\partial\left(x, v_{h}\right)}{\partial(s, t)}\right) J^{-1} d s d t \\
l\left(v_{h}\right)=\int_{\Sigma}(f \circ \mathscr{P})\left(v_{h} \circ \mathscr{P}\right) J d s d t
\end{gathered}
$$

where

$$
\frac{\partial(f, g)}{\partial(s, t)}=\frac{\partial f}{\partial s} \frac{\partial g}{\partial t}-\frac{\partial f}{\partial t} \frac{\partial g}{\partial s} .
$$

The integral in (13) is a defective, if $\mathscr{P}(s, t)$ has singularities. However, it converges when $\mathscr{P}(s, t)$ is an $H^{1}$-parameterization. Here, this integral is evaluated using the $G p \times G p$ points tensor-product Gaussian quadrature on each cell of $\mathcal{Q}_{h}$. To guarantee the accuracy of the numerical integral, we take as many Gaussian points as possible in the following experiments. Moreover, in these experiments, we take

$$
\mathcal{V}^{h}=\left\{H \circ \mathscr{P}^{-1}: H(s, t) \in \mathscr{H}_{h}^{3}\left(\mathcal{Q}_{h}\right),\left.H\right|_{\partial \Sigma}=0\right\} .
$$

By these a priori estimates (11) and (12), in the FEM, there is positive correlation between the convergence rate of numerical solutions and the regularity of the weak solution. Especially, when the weak solution $u \in H^{4}(\Omega)$, the optimal convergence rates with the $L^{2}(\Omega)$-norm and the $H^{1}(\Omega)$-seminorm are reached in the case of $p=3$. In the following section, we find that the optimal convergence rates are also reached, even if $u \in H^{\sigma}(\Omega), 1<\sigma<2$, by choosing a proper singular parameterization.

\subsection{Experiments with controllable singular parameterizations}

In this section, we discuss the convergence rates with the $L^{2}(\Omega)$-norm and the $H^{1}(\Omega)$-seminorm by introducing a controllable singular parameterization at the corner points. The controllable singular parameterization $\mathscr{P}_{\delta}(s, t)$ is defined as:

$$
\begin{aligned}
\mathscr{P}_{\delta}: \Sigma \longrightarrow \Omega \\
(s, t) \mapsto\left(s^{\delta} \cos t, s^{\delta} \sin t\right)
\end{aligned}
$$

where the Jacobian

$$
J=s^{2 \delta-1} .
$$

When $\delta=1, \mathscr{P}_{1}(s, t)$ has been used to overcome the loss of accuracy encountered when applying the standard schemes to two-dimensional elliptic problems in the crack problems in [18]. For $\mathscr{P}_{\delta}(s, t)$, the singularity can be controlled by different values of $\delta$. In addition, in Lemma 3.1, we prove that $\mathscr{P}_{\delta}$ is an $H^{1}$-parameterization. 
Lemma 3.1. If $H(s, t) \in \mathscr{H}_{h}^{3}(\Sigma)$ and $\left.H(s, t)\right|_{\partial \Sigma}=0$, then

$$
H \circ \mathscr{P}_{\delta}^{-1} \in H^{1}(\Omega), \forall i=1,2, \cdots, n .
$$

Proof. Denote $\mathscr{P}_{\delta}(s, t)=(x(s, t), y(s, t))=\left(s^{\delta} \cos t, t^{\delta} \sin t\right)$, where $(s, t) \in[0,1] \times$ $[0,1]$. Its Jacobian is $J=s^{2 \delta-1}$. In the following, we will check that, for each $H(s, t)$, the $L^{2}$-norm and the $H^{1}$ semi-norm are bounded. On the one hand,

$$
\left\|H \circ \mathscr{P}_{\delta}^{-1}\right\|_{L^{2}(\Omega)}^{2}=\int_{\Omega}\left(H \circ \mathscr{P}_{\delta}^{-1}\right)^{2} d x d y=\int_{\Sigma}(H(s, t))^{2} s^{2 \delta-1} d s d t .
$$

Then, $\left\|H \circ \mathscr{P}_{\delta}^{-1}\right\|_{L^{2}(\Omega)}$ is bounded. On the other hand,

$$
\begin{aligned}
\left|H \circ \mathscr{P}_{\delta}^{-1}\right|_{H^{1}(\Omega)}^{2} & =\int_{\Omega}\left(\left(\frac{\partial H \circ \mathscr{P}_{\delta}^{-1}}{\partial x}\right)^{2}+\left(\frac{\partial H \circ \mathscr{P}_{\delta}^{-1}}{\partial y}\right)^{2}\right) d x d y \\
& =\int_{[0,1] \times[0,1]} \frac{\left(\frac{\partial H}{\partial s}, \frac{\partial H}{\partial t}\right) \hat{J} \hat{J}^{T}\left(\frac{\partial H}{\partial s}, \frac{\partial H}{\partial t}\right)^{T}}{J} d s d t \\
& =\int_{[0,1] \times[0,1]} \frac{s^{2} \frac{\partial H}{\partial s}+\delta^{2} \frac{\partial H}{\partial t}}{s} d s d t
\end{aligned}
$$

where $\hat{J}=\left(\begin{array}{cc}s^{\delta} \cos t & s^{\delta} \sin t \\ -\delta s^{\delta-1} \sin t & \delta s^{\delta-1} \cos t\end{array}\right)$

Moreover, $s \mid H(s, t)$ because $\left.H(s, t)\right|_{\partial \Sigma}=0$. So, $s \mid \frac{\partial H}{\partial t}$. Thus, $\left|H \circ \mathscr{P}^{-1}\right|_{H^{1}(\Omega)}$ is bounded. In conclusion, $H \circ \mathscr{P}_{\delta}^{-1} \in H^{1}(\Omega)$.

By this controllable singular $H^{1}$-parameterization, we discuss the optimal convergence rates with $L^{2}(\Omega)$-norm and $H^{1}(\Omega)$-seminorm on a physical domain with corners such as the one shown in Figure 2. To discuss the regularity of weak solution cases differently, $\mathscr{P}_{\delta}$ is rewritten as

$$
\begin{aligned}
\mathscr{P}_{\delta, \alpha}:[0,2] & \times[0,2] \longrightarrow \Omega_{\alpha} \\
(s, t) & \mapsto\left(\left(\frac{s}{2}\right)^{\delta} \cos \left(\frac{\alpha \pi}{2} t\right),\left(\frac{s}{2}\right)^{\delta} \sin \left(\frac{\alpha \pi}{2} t\right)\right),
\end{aligned}
$$

where the included angle of $\Omega_{\alpha}$ is $\alpha \pi, \Omega=\mathscr{P}_{\delta}(\Sigma)$. In Examples 3.1 and 3.2, we discuss physical domains with $\alpha=2$ and $\alpha=3 / 2$, respectively.

Example 3.1. [ $\alpha=2]$ The elliptic boundary value problem:

$$
\begin{aligned}
& -\Delta u=\frac{3}{4}\left(x^{2}+y^{2}\right)^{-\frac{5}{4}}\left(1+\frac{5}{3} \sqrt{x^{2}+y^{2}}\right) y \text { in } \Omega_{2}, \\
& u=0 \text { on } \partial \Omega_{2},
\end{aligned}
$$




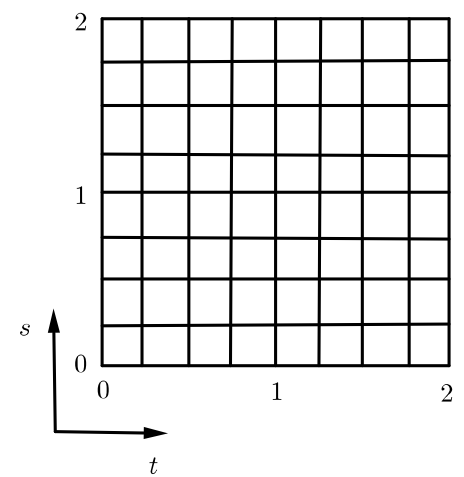

(a) The parametric domain $\Sigma$

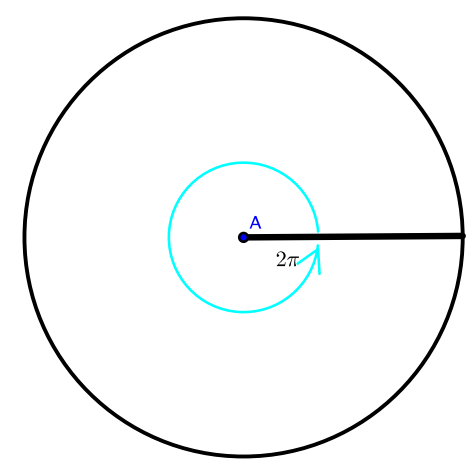

(b) The physical domain $\Omega_{2}$

Figure 4: The parametric domain and physical domain of Example 3.1

where the physical domain is shown in Figure $4(b)$ is

$$
\Omega_{2}=\left\{(x, y) \in \mathbb{R}^{2}: x=r \cos (\theta), y=r \sin (\theta), 0<r<1,0<\theta<2 \pi\right\} .
$$

The exact weak solution of this elliptic boundary value problem is

$$
u(x, y)=\left(x^{2}+y^{2}\right)^{-1 / 4}\left(1-\sqrt{x^{2}+y^{2}}\right) y
$$

and

$$
u \circ \mathscr{P}_{\delta, \alpha}(s, t)=\left(\frac{s}{2}\right)^{\delta / 2}\left[1-\left(\frac{s}{2}\right)^{\delta}\right] \sin \left(\frac{\alpha \pi}{2} t\right) .
$$

Thus,

$$
u \circ \mathscr{P}_{\delta, \alpha} \in H^{\frac{\delta}{2}+1-\epsilon}\left(\Sigma_{s}\right)(\forall \epsilon>0) ; u \circ \mathscr{P}_{\delta, \alpha} \in H^{4}\left(\Sigma_{r}\right) ; u \circ \mathscr{P}_{\delta, \alpha} \in W_{\infty}^{\frac{\delta}{2}-\epsilon}\left(\Sigma_{s}\right) .
$$

By Theorems 2.3 and 2.4, we have

Corollary 3.2. The $L^{2}(\Omega)$-norm approximation order is not less than $\min \left\{\left[\frac{3 \delta}{2}+\frac{1}{2}-\right.\right.$ $\epsilon], 4\}$; the $H^{1}(\Omega)$-seminorm approximation order is not less than $\min \left\{\left[\frac{\delta}{2}-1-\epsilon\right], 3\right\}$. Furthermore, when $\delta>\frac{7}{3}$, the optimal $L^{2}(\Omega)$-norm approximation order is reached; when $\delta>8$, the optimal $H^{1}(\Omega)$-seminorm approximation order is reached.

In the following, the convergence rates between the numerical solution and the exact solution of (16) are presented. In Figure 5(a), we present the numerical result with the $L^{2}(\Omega)$-norm by taking $\delta=2.4$. The optimal $L^{2}(\Omega)$ approximation order is reached by Corollary 3.2 because $\delta>7 / 3$. From the numerical result in Figure 5(a), the optimal convergence rate (i.e., 4) for solving the elliptic boundary value problem (16) is reached. 


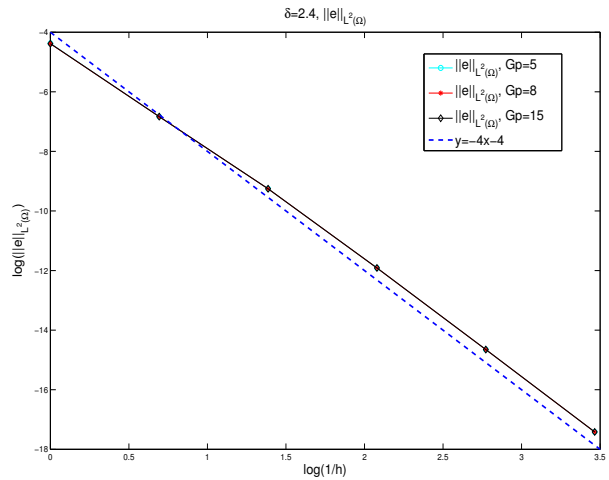

(a) $\delta=2.4, L^{2}(\Omega)$-norm

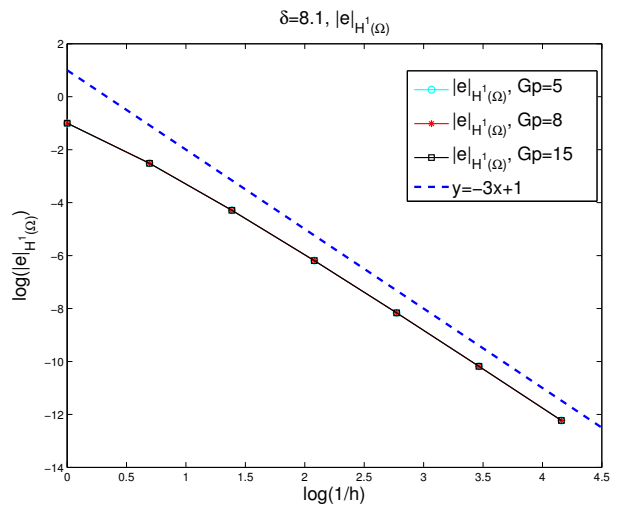

(b) $\delta=8.1, H^{1}(\Omega)$-seminorm

Figure 5: Errors with the $L^{2}(\Omega)$-norm and the $H^{1}(\Omega)$-seminorm for solving the elliptic boundary value problem (16)

Taking $\delta=8.1$, the optimal $H^{1}(\Omega)$-seminorm approximation order (i.e., 3) is obtained because $\delta=8.1>8$. The numerical results are presented in Figure 5(b). In Figure 5(b), the optimal convergence rate (i.e., 3) for solving the elliptic boundary value problem (16) is reached.

Example 3.2. $(\alpha=3 / 2)$ The elliptic boundary value problem:

$$
\begin{aligned}
& -\Delta u=2 x y\left(\frac{32}{9}\left(x^{2}+y^{2}\right)^{-5 / 3}-\frac{11}{9}\left(x^{2}+y^{2}\right)^{-7 / 6}\right) \text { in } \Omega, \\
& u=0 \quad \text { on } \partial \Omega
\end{aligned}
$$

where the physical domain shown in Figure 6(b) is

$$
\Omega=\left\{(x, y) \in \mathbb{R}^{2}: x=r \cos \theta, y=r \sin \theta, 0<r<1,0<\theta<\frac{3}{2} \pi\right\} .
$$

The exact solution of this elliptic boundary value problem is

$$
\begin{gathered}
u(x, y)=2 x y\left(x^{2}+y^{2}\right)^{-2 / 3}\left(1-\sqrt{x^{2}+y^{2}}\right) . \\
u \circ \mathscr{P}_{\delta, \alpha}(s, t)=\left[\left(\frac{s}{2}\right)^{\frac{2 \delta}{3}}\left(1-\left(\frac{s}{2}\right)^{\delta}\right)\right] \sin (\alpha \pi t) \in H^{\sigma}(\Omega)(1<\sigma<2)
\end{gathered}
$$

Thus $\forall \epsilon>0$,

$$
u \circ \mathscr{P}_{\delta, \alpha} \in H^{\frac{2 \delta}{3}+1-\epsilon}\left(\Sigma_{s}\right) ; u \circ \mathscr{P}_{\delta, \alpha} \in H^{4}\left(\Sigma_{r}\right) ; u \circ \mathscr{P}_{\delta, \alpha} \in W_{\infty}^{\frac{2 \delta}{3}-\epsilon}\left(\Sigma_{s}\right)
$$

By Theorems 2.3 and 2.4, we obtain the following

Corollary 3.3. The $L^{2}(\Omega)$-norm approximation order is not less than min $\left\{\left[\frac{5 \delta}{3}+\right.\right.$ $\left.\left.\frac{1}{2}\right], 4\right\}$, and the $H^{1}(\Omega)$-norm approximation order is not less than $\min \left\{\left[\frac{2 \delta}{3}-1-\epsilon\right], 3\right\}$. When $\delta>2.1$, the optimal $L^{2}(\Omega)$-norm approximation order is reached. When $\delta>6$, the optimal $H^{1}(\Omega)$-seminorm approximation order is reached. 


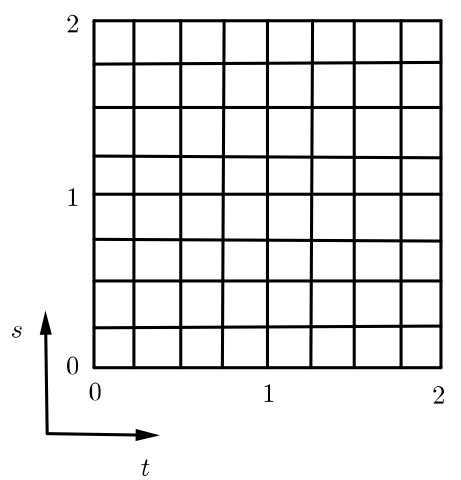

(a) The parametric domain $\Sigma$

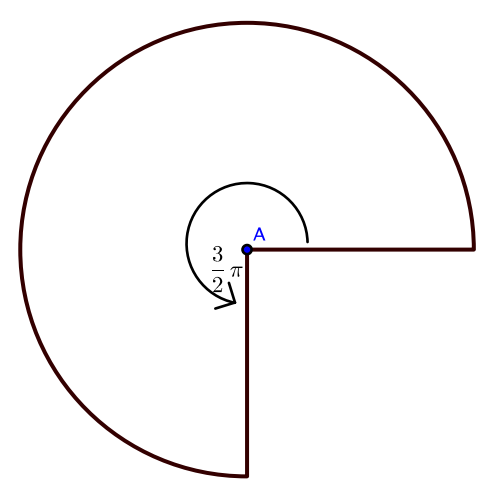

(b) The physical domain $\Omega_{\frac{3}{2}}$

Figure 6: The parametric domain and physical domain of Example 3.2

From Corollary 3.3, when we take $\delta=2.2$, the $L^{2}(\Omega)$-norm approximation order is optimal (i.e., 4) because $\delta>2.1$. In Figure 7(a), we present the numerical results of errors with the $L^{2}(\Omega)$-norm by $\delta=2.2$. By this numerical result, the optimal $L^{2}(\Omega)$ norm convergence rate is reached.

Taking $\delta=6.1$, the optimal $H^{1}(\Omega)$-seminorm approximation order (i.e. 3) is reached because $\delta>6$. In Figure $7(b)$, the numerical errors with the $H^{1}(\Omega)$-seminorm for solving the elliptic boundary value problem (17) are presented.

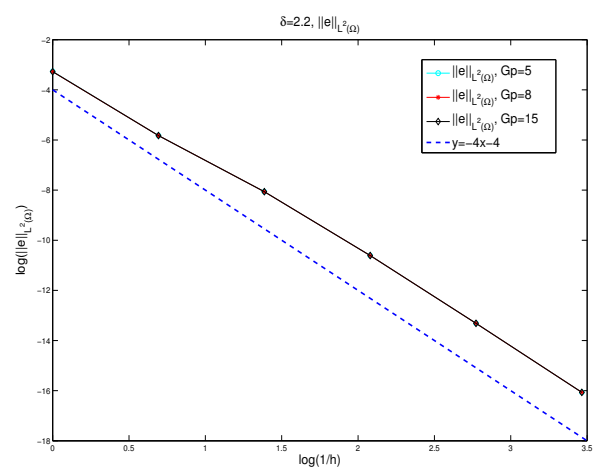

(a) $\delta=2.2, L^{2}(\Omega)$-norm

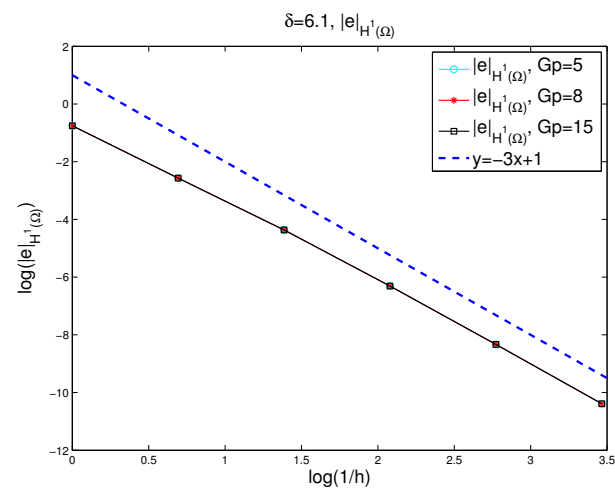

(b) $\delta=6.1, H^{1}(\Omega)$-seminorm

Figure 7: Errors with the $L^{2}(\Omega)$-norm and $H^{1}(\Omega)$-seminorm in Example 3.2

\subsection{Experiments with singular parameterizations in isogeometric analysis}

In this section, after introducing parameterizations in the IGA as background, we solve the elliptic boundary value problem (8) and compare the results by the IGA with the ones by the FEM with $\mathscr{H}_{h}^{3}\left(\mathcal{Q}_{h}\right)$ as test functions and trial functions. In addition, 
the FEM is a special case of the IGA method, where the parameterization is an identity map, which is a non-singular parameterization.

In the IGA, the geometric description and numerical analysis share the same basis functions. Thus, in this section, parameterizations are represented by splines in $\mathscr{H}_{h}^{3}\left(\mathcal{Q}_{h}\right)$, i.e.,

$$
\mathscr{P}(s, t)=(x(s, t), y(s, t))^{T}=\sum_{i=1}^{n} \mathbf{P}_{i} H_{i}(s, t),
$$

where $\left\{H_{1}(s, t), H_{2}(s, t), \cdots, H_{n}(s, t)\right\}$ is a set of basis functions of $\mathscr{H}_{h}^{3}\left(\mathcal{Q}_{h}\right)$ and $\mathbf{P}_{i} \in$ $\mathbb{R}^{2}$.

Corollary 3.4. If $u \circ \mathscr{P} \in H^{4}\left(\Sigma_{r}\right), u \circ \mathscr{P} \in H^{k^{\prime}+1}\left(\Sigma_{s}\right)$, and $J \sim h^{\sigma}$ on singular cells, then

$$
\| u-\left.\Pi_{\mathscr{H}_{h}^{3}}^{\hat{A}} u\right|_{L^{2}(\Omega)} \leq C_{1} h^{4}|u \circ \mathscr{P}|_{H^{4}\left(\Sigma_{r}\right)}+C_{2} h^{k^{\prime}+1+\sigma / 2}|u \circ \mathscr{P}|_{H^{k^{\prime}+1}\left(\Sigma_{s}\right)} ;
$$

In the FEM, if $u \in H^{4}(\Omega)$ the optimal convergence rate is reached. However, if $u \in H^{4-\sigma / 2}\left(\Omega_{s}\right)$ and $u \in H^{4}\left(\Omega_{r}\right)$, then the approximation error order is optimal by using the singular parameterization $\mathscr{P}(s, t)$, where $\Omega_{s}=\mathscr{P}\left(\Sigma_{s}\right)$ and $\Omega_{t}=\mathscr{P}\left(\Sigma_{t}\right)$.

\subsubsection{Numerical results}

In the following, we present the numerical results in Examples 3.3 and 3.4 and analyze these results in Section 3.3.2. In these examples, the singular parameterization $\mathscr{P}(s, t)$ satisfies the integrability assumption 5.1 in [9] and its construction method follows [20]. By its construction, its Jacobian on a singular cell is $J \sim h^{2}$, i.e. $\sigma=2$ in Corollary 3.4.

Example $3.3(\alpha=2)$. The parametric mesh is shown in Figure 8(a). The parameterization is presented in Figure 8(b). In the following, we present the numerical results.

(a). If the exact solution is

$$
u_{a}(x, y)=\left(4-x^{2}\right)\left(4-y^{2}\right) \sin y
$$

then the numerical convergence rates within the IGA framework with the singular parameterization and FEM are shown in Figures 9(a) and 9(b).

(b). If the exact solution is

$$
u_{b}(x, y)=\frac{y\left(4-x^{2}\right)\left(4-y^{2}\right)}{\left(x^{2}+y^{2}\right)^{1 / 4}}
$$

then the numerical convergence rates within the IGA framework with the singular parameterization and FEM are shown in Figures 10(a) and 10(b).

(c). If the exact solution is

$$
u_{c}(x, y)=y\left(x^{2}+y^{2}\right)^{1 / 4}\left(4-x^{2}\right)\left(4-y^{2}\right),
$$

then the numerical convergence rates within the IGA framework with the singular parameterization and FEM are shown in Figures 11(a) and 11(b). 


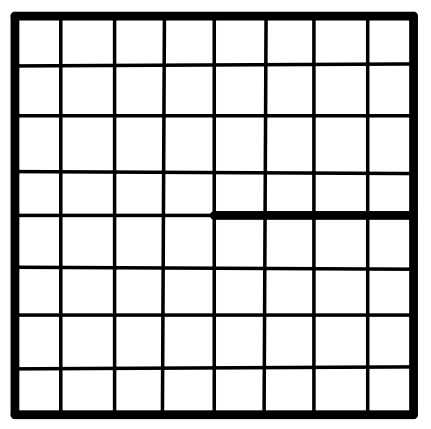

(a) The parametric mesh

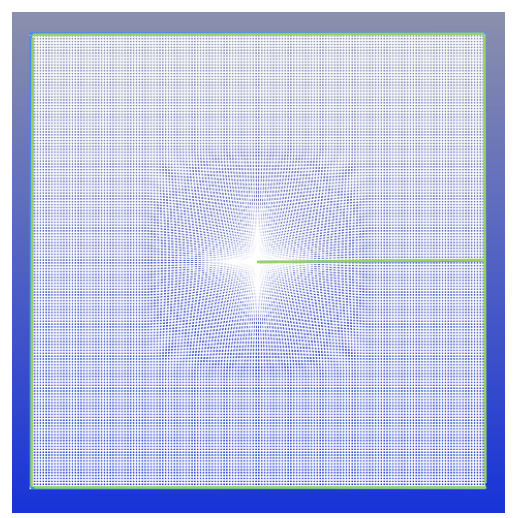

(b) The parameterization with a singularity

Figure 8: The parametric domain and physical domain of Example 3.3

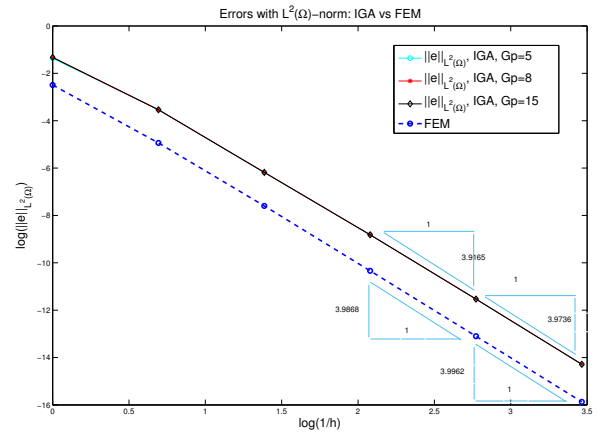

(a) $L^{2}(\Omega)$-norm

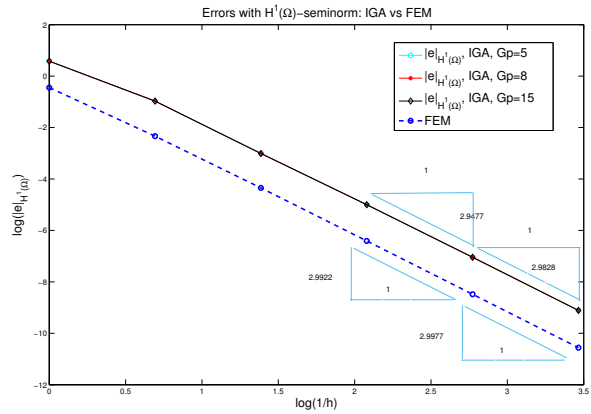

(b) $H^{1}(\Omega)$-seminorm

Figure 9: The errors with the $L^{2}(\Omega)$-norm and the $H^{1}(\Omega)$-seminorm of Example 3.3 (a)

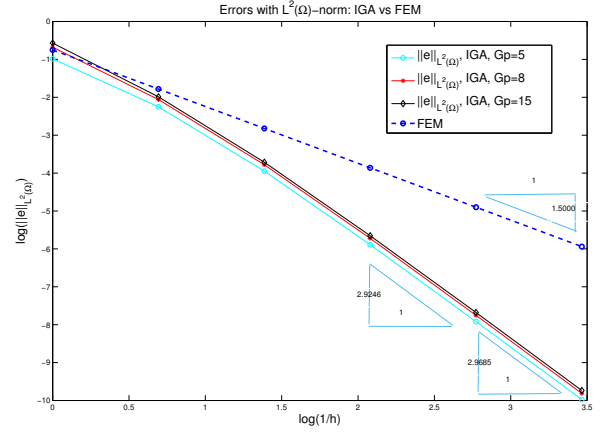

(a) $L^{2}(\Omega)$-norm

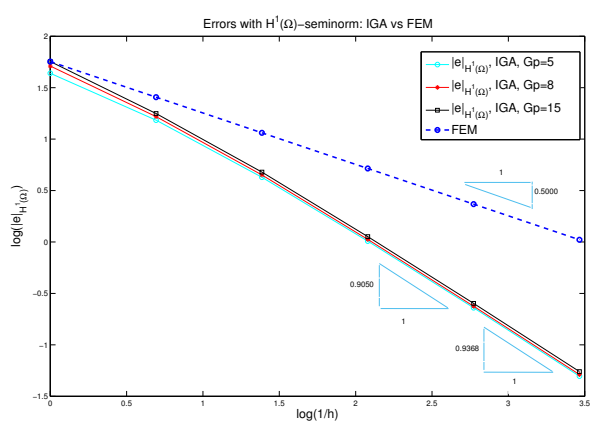

(b) $H^{1}(\Omega)$-seminorm

Figure 10: The errors with the $L^{2}(\Omega)$-norm and the $H^{1}(\Omega)$-seminorm of Example 3.3 (b) 


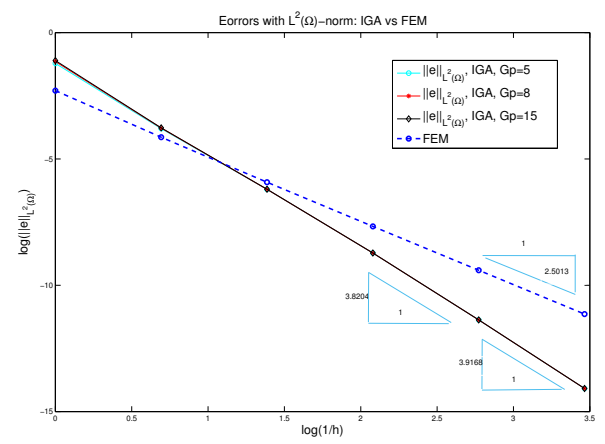

(a) $L^{2}(\Omega)$-norm

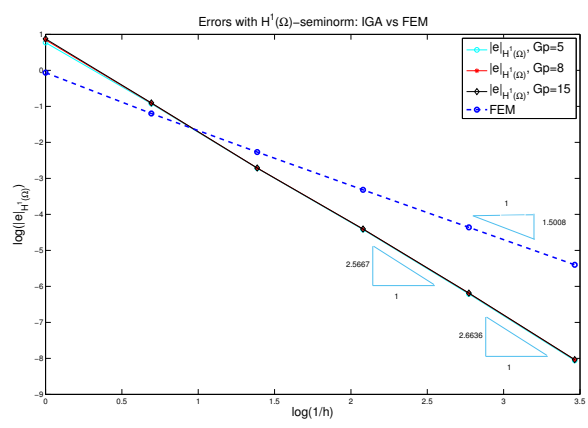

(b) $H^{1}(\Omega)$-seminorm

Figure 11: The errors with the $L^{2}(\Omega)$-norm and the $H^{1}(\Omega)$-seminorm of Example 3.3 (c)

Example $3.4(\alpha=3 / 2)$. The parametric mesh is shown in Figure 12(a). The parameterization is presented in Figure 12(b). In the following, we present the numerical results.

(a). If the exact solution is

$$
u_{a}(x, y)=\left(1-x^{2}\right)\left(1-y^{2}\right) x^{3} y^{2}
$$

then the numerical convergence rates within the IGA framework with the singular parameterization and FEM are shown in Figures 13(a) and 13(b).

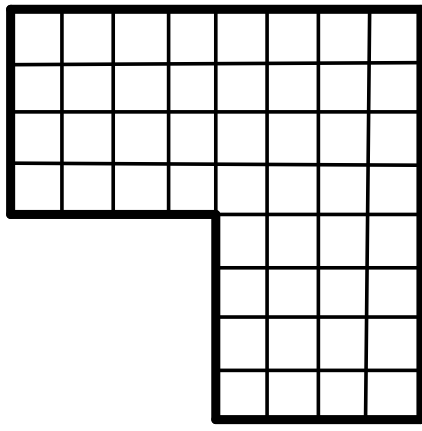

(a) The parametric mesh

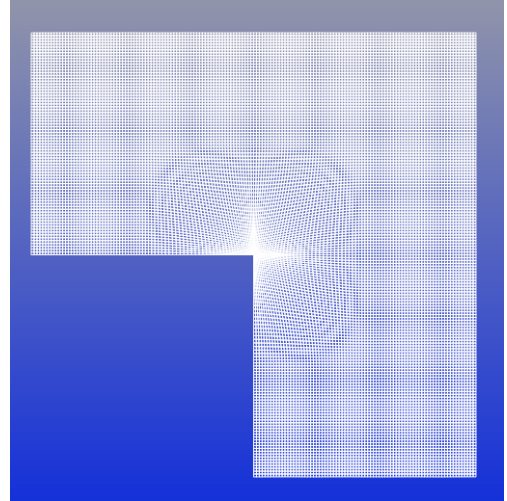

(b) The parameterization with a singularity

Figure 12: The parametric domain and physical domain of Example 3.4

(b). If the exact solution is

$$
u_{b}(x, y)=\left(1-x^{2}\right)\left(1-y^{2}\right) x y /\left(x^{2}+y^{2}\right)^{2 / 3},
$$

then the numerical convergence rates within IGA framework with the singular parameterization and FEM are shown in Figures $14(a)$ and $14(b)$. 


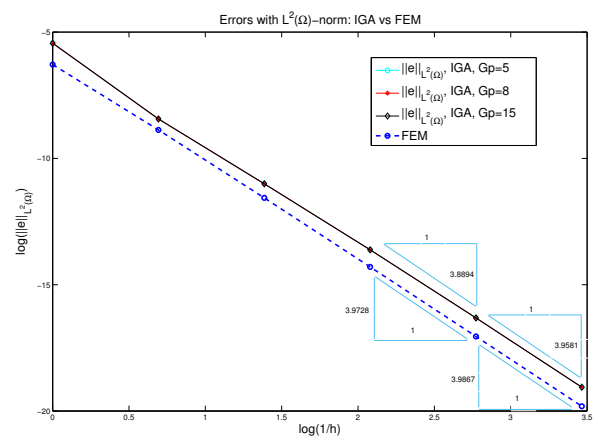

(a) $L^{2}(\Omega)$-norm

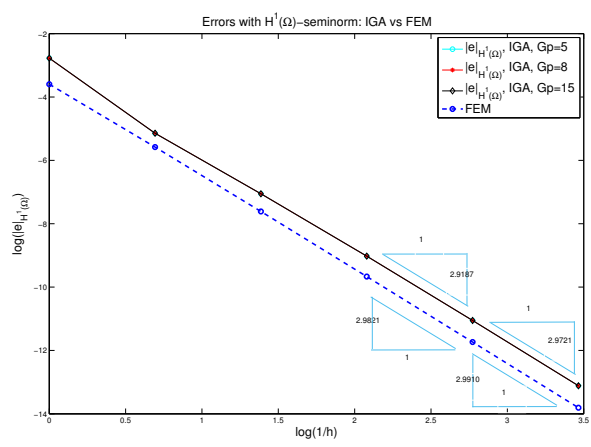

(b) $H^{1}(\Omega)$-seminorm

Figure 13: Errors with the $L^{2}(\Omega)$-norm and the $H^{1}(\Omega)$-seminorm in Example 3.4(a)

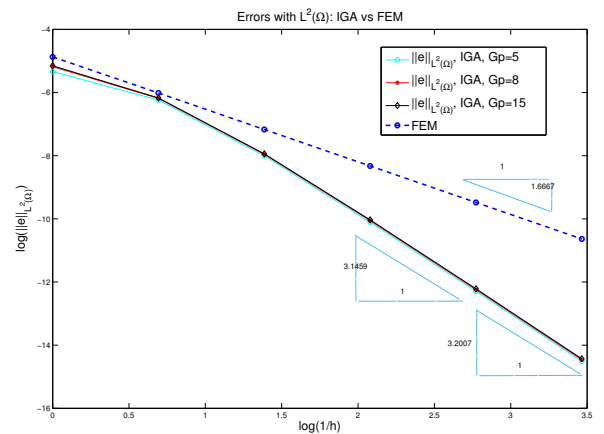

(a) $L^{2}(\Omega)$-norm

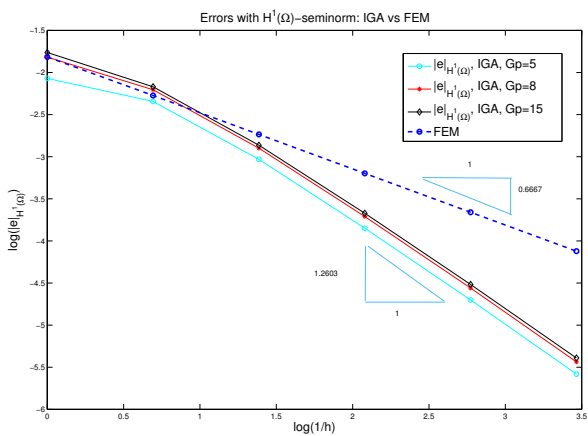

(b) $H^{1}(\Omega)$-seminorm

Figure 14: Errors with the $L^{2}(\Omega)$-norm and the $H^{1}(\Omega)$-seminorm in Example 3.4(b)

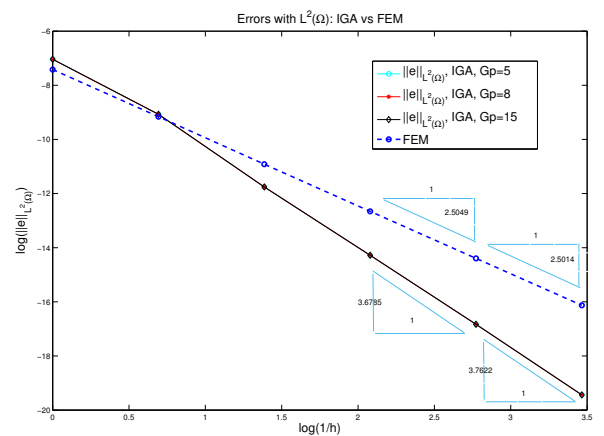

(a) $L^{2}(\Omega)$-norm

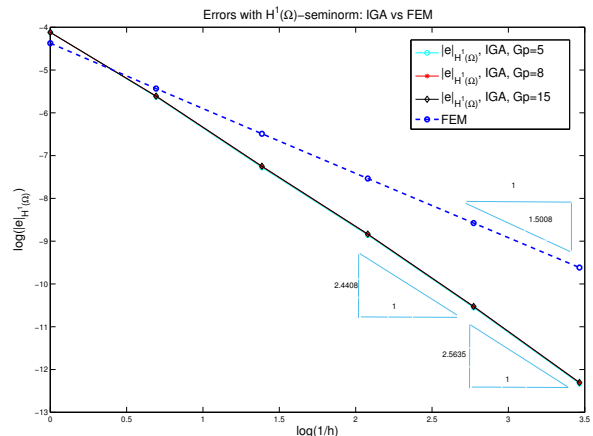

(b) $H^{1}(\Omega)$-seminorm

Figure 15: Errors with the $L^{2}(\Omega)$-norm and the $H^{1}(\Omega)$-seminorm in Example 3.4(c) 
(c). If the exact solution is

$$
u_{c}(x, y)=\left(x^{2}+y^{2}\right)^{-1 / 4} x y\left(1-x^{2}\right)\left(1-y^{2}\right),
$$

then the numerical convergence rates within the IGA framework with the singular parameterization and FEM are shown in Figures 15(a) and 15(b).

3.3.2. Discussion of the numerical results in Examples 3.3 and 3.4:

1. For smooth weak solutions, the FEM and the IGA with singular parameterizations are reached optimal convergence rates. In Examples 3.3(a) and 3.4(a), $u_{a}(x, y) \in$ $H^{4}(\Omega), 4$ for the $L^{2}(\Omega)$-norm and 3 for the $H^{1}(\Omega)$-seminorm are reached in Figures $9(\mathrm{a}), 13(\mathrm{a})$ and $9(\mathrm{~b}), 13(\mathrm{~b})$.

2. For non-smooth weak solutions, the convergence rates by IGA with singular parameterizations are better than the ones by the FEM. In Examples 3.3(b) and 3.3(c) (or Examples 3.4(b) and 3.4(c)), $u_{b}(x, y), u_{c}(x, y) \in \mathcal{F}(\Omega)$ but $u_{b}(x, y), u_{c}(x, y)$ are not in $H^{4}(\Omega)$. Although $u_{b}(x, y)$ and $u_{c}(x, y)$ have different regularities, in Figures 10(a) and 11(a) (or Figures 14(a) and 15(a)), the convergence rates with the $L^{2}(\Omega)$-norm by IGA with singular parameterizations are better than the ones by the FEM. There is a similar result with the $H^{1}(\Omega)$-seminorm in Figures 10(b) and 11(b) (or Figures 14(b) and $15(\mathrm{~b}))$.

\section{Conclusions and the Future Work}

In this paper, we discuss the convergence rates with singular parameterizations for solving elliptic boundary value problems in the IGA. To discuss all possible weak solutions of elliptic boundary value problems with smooth coefficients, this work is based on a weak solution space $\mathcal{F}(\Omega)$. The approximation errors on $\mathcal{F}(\Omega)$ with the $L^{2}(\Omega)$-norm and the $H^{1}(\Omega)$-seminorm are estimated locally, i.e., these errors reflect the impact of singularities of parameterizations. Based on this approximation error estimates, in Section 3, the convergence rates with the $L^{2}(\Omega)$-norm and the $H^{1}(\Omega)$-seminorm are discussed with a controllable singular parameterization and singular parameterizations in the IGA. By a controllable singular parameterization, although the optimal convergence rates can not be reached by the FEM, the optimal ones are reached by choosing proper singularities in the IGA framework. Later, we compare the convergence rates by singular $H^{1}$-parameterizations in the IGA and the ones by the FEM. For smooth weak solutions by the FEM and the IGA with the singular parameterizations, both of them obtain the optimal convergence rates; for non-smooth weak solutions by these two methods, the results by the IGA with the singular parameterizations are better than the ones by the FEM. In the future, we will consider the behaviour of singular parameterizations for solving higher order partial differential equations.

\section{Reference}

[1] T. J. R. Hughes, J. A. Cottrell and Y. Bazilevs. "Isogeometric analysis: CAD, finite elements, NURBS, exact geometry and mesh refinement". Computer Methods in Applied Mechanics and Engineering, vol. 194, Issues. 39-41, p. 4135-4195, 2005. 
[2] Y. Bazilevs, L. B. da Veiga, J. A. Cottrell, T. J. R. Hughes and G. Sangalli. Isogeometric analysis: approximation, stability and error estimates for h-refined meshes. Mathematical Models and Methods in Applied Sciences, vol. 16, p. 10311090, 2006.

[3] A. Tagliabue, L. Dedé and A. Quarteroni. Isogeometric Analysis and error estimates for high order partial differential equations in fluid dynamics Computers \& Fluids, vol. 102, p. 277-303, 2014.

[4] E. Cohen, T. Martin, R. Kirby, T. Lyche and R. Riesenfeld. Analysis-aware modeling: Understanding quality considerations in modeling for isogeometric analysis. Computer Methods in Appiled Mechanics and Engineering, vol. 199, p. 334-356, 2010.

[5] G. Xu, B. Mourrain, R. Duvigneau and A. Galligo. Constructing analysis-suitable parameterization of computational domain from CAD boundary by variational harmonic method. Journal of Computational Physics, vol. 252, p. 275-289, 2013.

[6] G. Xu, B. Mourrain, R. Duvigneau and A. Galligo. Parameterization of computational domain in isogeometric analysis: Methods and comparison. Computer Methods in Applied Mechanics and Engineering, vol. 200, p. 2021-2031, 2011.

[7] J. Lu. "Circular element: Isogeometric elements of smooth boundary'. Computer Methods in Applied Mechanics and Engineering, vol. 198, p. 2391-2402, 2009.

[8] M. Wu, B. Mourrain, A. Galligo and B. Nkonga. Hermite type spline spaces over rectangular meshes with arbitrary topology accepted by Communications in Computational Physics. https://hal.inria.fr/hal-01196428v3/document, version 3, 2016.

[9] T. Takacs, B. Jüttler. "Existence of Stiffness Matrix Integrals for Singularly Parameterized Domains in Isogeometric Analysis". Computer Methods in Applied Mechanics and Engineering, vol. 200, p. 3568-3582, 2011.

[10] T. Takacs, B. Jüttler. " $H^{2}$ regularity properties of singular parameterzations in isogeometric analysis". Graphical Models, vol. 74, p. 361-372, 2012.

[11] T. Takacs. Approximation properties of isogeometric function spaces on singularly parameterized domains preprint, https://arxiv.org/abs/1507.08095.

[12] T. Takacs. Construction of smooth isogeometric function spaces on singularly parameterized domains. Curves and Surfaces 2014, LNCS 9213, DOI: 10.1007/9783-319-22804-4 30, p. 433-451, 2015.

[13] J. W. Jeong, H.-S. Oh, S. Kang and H. Kim. "Mapping techniques for isogeometric analysis of elliptic boundary value problems containing singularities". Computer Methods in Applied Mechanics and Engineering, vol. 254, p. 334-352, 2013. 
[14] H.-S. Oh, H. Kim and J. W. Jeong. "Enriched isogeometric analysis of elliptic boundary value problems in domains with cracks and/or corners". International Journal for Numerical Methods in Engineering, vol. 97, p. 149-180, 2014.

[15] G. Strang and G. Fix. An analysis of the finite element method Prentice Hall, New-York, 1973.

[16] J. Deng, F. Chen, and Y. Feng. Dimensions of spline spaces over T-meshes. Journal of Computational and Applied Mathematics, vol. 194, p. 267-283, 2006.

[17] G. Constantine and T. Savits. A multivariate faa di bruno formula with applications Transactions of the American Mathematical Society, vol. 348, p. 503-520, 1996.

[18] Z. Yosibash and B. Schiff. Superelements for the finite element solution of twodimensional elliptic problems with boundary singularities Finite Elements in Analysis and Design, vol. 26, p. 315-335, 1997.

[19] P. G. Ciarlet, P. A. Raviart. General lagrange and hermit interpolation in $\mathbb{R}^{n}$ with applications to finite element methods, Archive for Rational Mechanics and Analysis, vol. 46, p. 177-199, 1972.

[20] M. Wu, B. Mourrain, A. Galligo, B. Nkonga. H1-parameterizations of planar physical domains with complex topology in isogeometric analysis., preprint, https://hal.inria.fr/hal-01196435/, 2015. 